\section{Exploratory, cognitive, and depressive- like behaviors in adult and pediatric mice exposed to controlled cortical impact}

\author{
Suk-Woo Lee ${ }^{1,2}$, Mun-Sun Jang ${ }^{2,3}$, Seong-Hae Jeong ${ }^{4}$, Hoon Kim ${ }^{1,2}$ \\ 1 Department of Emergency Medicine, Chungbuk National University Hospital, Cheongju, Korea \\ ${ }^{2}$ Department of Emergency Medicine, Chungbuk National University College of Medicine, Cheongju, Korea \\ ${ }^{3}$ Department of Emergency Medical Technology, Chungbuk Health \& Science University, Cheongju, Korea \\ ${ }^{4}$ Department of Neurology, Chungnam National University College of Medicine, Daejeon, Korea
}

Objective Sequelae of behavioral impairments associated with human traumatic brain injury (TBI) include neurobehavioral problems. We compared exploratory, cognitive, and depressive-like behaviors in pediatric and adult male mice exposed to controlled cortical impact (CCI).

Methods Pediatric ( 21 to 25 days old) and adult ( 8 to 12 weeks old) male C57BI/6 mice underwent $\mathrm{CCl}$ at a 2-mm depth of deflection. Hematoxylin and eosin staining was performed 3 to 7 days after recovery from $\mathrm{CCl}$, and injury volume was analyzed using ImageJ. Neurobehavioral characterization after $\mathrm{CCl}$ was performed using the Barnes maze test (BMT), passive avoidance test, open-field test, light/dark test, tail suspension test, and rotarod test. Acutely and subacutely ( 3 and 7 days after $\mathrm{CCl}$, respectively), $\mathrm{CCl}$ mice showed graded injury compared to sham mice for all analyzed deflection depths.

Results Time-dependent differences in injury volume were noted between 3 and 7 days following 2-mm TBI in adult mice. In the BMT, 2-mm TBI adults showed spatial memory deficits compared to sham adults $(\mathrm{P}<0.05)$. However, no difference in spatial learning and memory was found between sham and 2-mm CCl groups among pediatric mice. The open-field test, light/ dark test, and tail suspension test did not reveal differences in anxiety-like behaviors in both age groups.

Conclusion Our findings revealed a graded injury response in both age groups. The BMT was an efficient cognitive test for assessing spatial/non-spatial learning following $\mathrm{CCl}$ in adult mice; however, spatial learning impairments in pediatric mice could not be assessed.

Keywords Brain injuries, traumatic; Controlled cortical impact; Age difference; Neurobehavioral differences
elSSN: 2383-4625

Received: 20 March 2018

Revised: 16 May 2018

Accepted: 17 May 2018

Correspondence to: Hoon Kim Department of Emergency Medicine, Chungbuk National University College of Medicine, 1 Chungdae-ro, Seowongu, Cheongju 28644, Korea

E-mail: nichekh2000@chungbuk.ac.kr ORCID

http://orcid.org/0000-0002-2133-0311

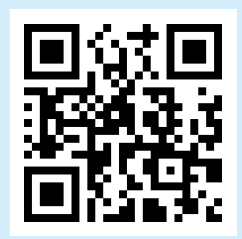

How to cite this article:

Lee SW, Jang MS, Jeong SH, Kim H. Exploratory, cognitive, and depressive-like behaviors in adult and pediatric mice exposed to controlled cortical impact. Clin Exp Emerg Med 2019;6(2):125-137.

This is an Open Access article distributed under the terms of the Creative Commons Attribution Non-Commercial License (http:// creativecommons.org/licenses/by-nc/4.0/). 


Capsule
Summary is already known
$\begin{aligned} & \text { Traumatic brain injury is a significant cause of morbidity and mortality in pediatric and adult populations. There are } \\ & \text { striking discrepancies in the literature in defining the age at which the prognosis worsens significantly. }\end{aligned}$
$\begin{aligned} & \text { What is new in the current study } \\ & \text { Differences in the behavior of pediatric and adult mice after traumatic brain injury were evaluated. A graded injury re- } \\ & \text { sponse was observed in both the evaluated age groups. Pediatric mice exhibited a faster progression of injury than adult } \\ & \text { mice. }\end{aligned}$

\section{INTRODUCTION}

Traumatic brain injury (TBI) is a significant cause of morbidity and mortality in pediatric and adult populations. ${ }^{1,2}$ In the United States, TBI affects more than 1.5 million people annually; approximately 300,000 are hospitalized and more than 50,000 die with $\mathrm{TBI}$ as a contributing cause, accounting for approximately one-third of injury-related deaths., ${ }^{3,4}$ Approximately 475,000 children aged 0 to 14 years are diagnosed with TBI annually in the United States and TBI is the current leading cause of death among children older than 1 year. ${ }^{5}$

Regardless of severity, several patients with TBI report some degree of neurobehavioral sequelae after injury. ${ }^{6}$ Significant changes in cognitive, behavioral, and emotional functioning are prevalent in patients with TBI. Cognitive complaints are typically reported in the areas of memory, attention, and executive functioning. ' Additionally, increased irritability, depression, or anxiety is often reported by individuals with TBI and other behavioral changes may include impulsive behaviors, reduced frustration tolerance, lack of empathy, emotional lability, apathy, or aggression. ${ }^{7}$ In particular, severe and moderate TBI frequently produces many types of temporary or permanent physical, cognitive, behavioral, and emotional impairments. ${ }^{8}$ Initial and persistent cognitive deficits after TBI are the most common complaints, including loss of short-term memory and learning impairment. Furthermore, severe and moderate TBI results in an increased relative risk of various psychiatric disorders, including mood and anxiety disorders. ${ }^{9}$ The lack of identification of neurobehavioral sequelae following TBI can lead to no or inappropriate treatment.

Unlike in adults, the pediatric brain undergoes rapid development. Therefore, pediatric neurotrauma is a frequent cause of neurobehavioral morbidity and can result in unique challenges as the consequences of injuries are overlaid on normal developmental changes. ${ }^{10}$ In addition, injury mechanisms in the pediatric population differ from those in adults, potentially resulting in unique primary and secondary lesions in the developing brain. ${ }^{11}$
Although many factors affect the clinical outcome of patients following TBI, patient age has been found to be one of the most reliable and consistent prognostic factors following $\mathrm{TBI}$ in various clinical studies, where older age is associated with worse out-

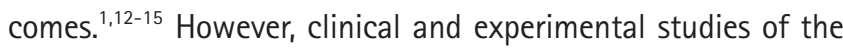
prognostic differences between the developing and adult brain following TBI are lacking. Knowledge of the age-specific post-injury neurobehavioral sequelae is essential for understanding and managing children with neurotrauma.

In a recent study, of age-dependent neuronal sensitivities to ischemic injury adult (8 to 12 weeks) and pediatric (postnatal 20 to 25 days) mice were subjected to 6-minute cardiac arrest and cardiopulmonary resuscitation; cardiac arrest produced global cerebral ischemia and neuronal injury in vulnerable brain regions in juvenile mice. ${ }^{16}$ Namely, the injured area and degree of injury was different from that in the adult brain. Similarly, the current study hypothesized that TBI has age-dependent neuronal sensitivity to traumatic injury. Therefore, an experimental approach to control various confounding factors is required to investigate the differences in vulnerability to TBI between the pediatric and adult brain.

Different experimental TBI models have been used to reflect the heterogeneous clinical situation in studies of TBI outcomes. Among TBI animal models, the controlled cortical impact (CCI) model can produce a more precise injury by controlling deformation parameters, such as time, velocity, and depth of impact, and thus, has advantages over other TBI models with respect to the absence of a risk for rebound injury, higher accuracy, reproducibility, and simplicity. ${ }^{17}$ There are limited published studies describing the differences in neurobehavioral outcomes between pediatric and adult patients after the same magnitude of TBI.

This study assessed the differences in exploratory, cognitive, and depressive-like behaviors between pediatric and adult male mice exposed to moderate to severe $\mathrm{CCl}$. Using neurobehavioral assessments evaluating outcomes after $\mathrm{TBI}$, we expect to (1) understand the effects of injury on cognitive skills, behavior, and 
learning; and (2) determine how these outcomes are related to the brain insult and any ensuing abnormalities in neural development.

\section{METHODS}

\section{Animal population}

All experimental protocols were approved by the animal care and use committee of Chungbuk National University, Cheongju, Korea (CBNUR-854-15). We used 8- to 12-week-old adult and 21 - to 25-day-old pediatric C57BI/6 male mice in this study. Mice were housed with a standard 12-hour light:dark cycle and had ad libitum access to food and water.

\section{Surgical procedures}

Mice were anesthetized with an intramuscular injection of 15 $\mathrm{mg} / \mathrm{kg}$ of tiletamine/zolazepam (Zoletil; Virbac, Carros, France). The head of the mouse was shaved between the ears and cleaned using betadine, a midline scalp incision was made, and the right parietal bone was exposed. Next, a 4-mm diameter circle was drawn centered between the lambda and bregma $0.5 \mathrm{~mm}$ right of the midline. A right parietal craniotomy was carefully performed using a surgical microscope and a micromotor drill
(Stoelting, Wood Dale, IL, USA) along the marked circle. Next, the $\mathrm{CCl}$ device was calibrated with respect to the exposed dura mater within the craniotomy. The parameters of the impact applied to the experimental animals were a depth of $2.0 \mathrm{~mm}$, mean velocity of $3.0 \mathrm{~m} / \mathrm{sec}$, and duration of $500 \mathrm{~ms}$. After the impact, the scalp incision was sutured with 5-0 nylon. The sham-operated groups received a craniotomy without $\mathrm{CCl}$ injury. The surgical procedure is shown in Fig. 1.

\section{Health assessment score}

Mice were weighed daily and a health assessment score was recorded for each mouse after surgery. The graded scoring systems ranged from 0 to 2,0 to 3 , or 0 to 5 depending on the behavior assessed, with 0 indicating no deficit and higher values indicating significant impairment. The behaviors assessed included consciousness (0-3), interaction (0-2), ability to grab a wire top (0$2)$, motor function (0-5), and activity (0-2). ${ }^{18,19}$ Scores in each category were summated to generate an overall health assessment score.

\section{Hematoxylin and eosin staining and estimations of corti- cal lesion volume}

At 3 and 7 days after TBI, animals were anesthetized with $15 \mathrm{mg} /$

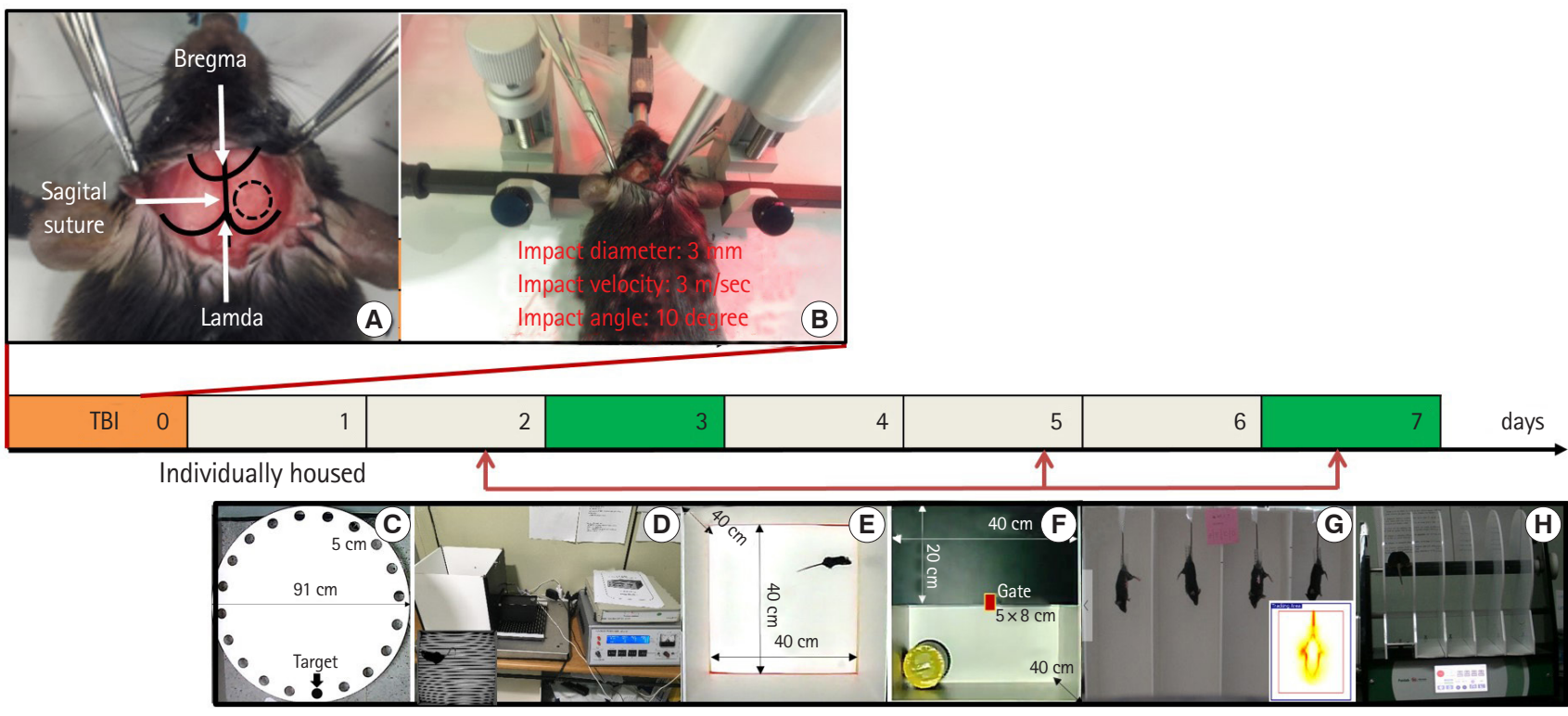

Fig. 1. Schematic timeline of experimental procedures in this study. The procedure for controlled cortical impact is illustrated in (A) and (B). At the beginning of surgery (day 0), the mouse head was stably fixed on a stereotactic frame with ear bar and mouth bits. The right skull was exposed, and a 4-mm circle was drawn in the middle between the bregma and lambda (A). The bone was removed by drilling to generate a window for impact. The impactor tip was retracted and lowered to the surface of the exposed dura until contact was made (B). Neurobehavioral tests were conducted to assess memory deficits, anxiety-like behaviors and motor performance (C-F). (C) Banes maze test (Acquisition trials, day 2-4; Probe trial, day 7). (D) Passiveavoidance test (Acquisition trials, day 3; Probe trial, day 7). (E) Locomotor activity test (day 7). (F) Light/dark test (day 7). (G) Tail suspension test (day 7). (H) Rota-rod test (day 7). Histological analysis was performed at day 3 and 7. 


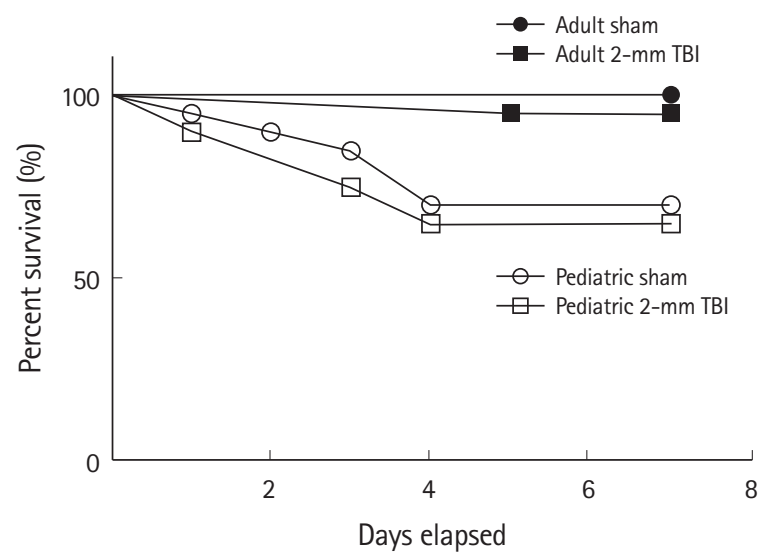

Fig. 2. Kaplan-Meier survival analysis of the four groups. Significantly improved survival was observed in the adult group compared with that in the pediatric group. The log rank statistic was 13.06. $\mathrm{P}<0.001$. TBI, traumatic brain injury.

$\mathrm{kg}$ of tiletamine/zolazepam and transcardially perfused with cold $0.9 \%$ saline followed by $10 \%$ formalin. Brains were removed, post-fixed with 10\% formalin, and embedded in paraffin. Coronal sections, 6- $\mu \mathrm{m}$ thick, were collected at 100-micron intervals and stained with hematoxylin and eosin (H\&E). Micrographs of the HEtE-stained sections were obtained to record and calculate the extent of injury at a magnification of $1.25 \times$ using a CX43 Olympus microscope (Olympus, Tokyo, Japan).

Cortical lesion volumes $\left(\mathrm{mm}^{3}\right)$ were determined by calculating the area of the lesion $\left(\mathrm{mm}^{2}\right)$ at a magnification of $\times 1.25$ using a CX43 microscope. This was done by outlining the missing cortical tissue for each section sliced at 500- $\mu \mathrm{m}$ intervals, and then multiplying the sum of lesion areas obtained from each section with the distance between sections. Lesion volumes were analyzed using ImageJ ver. 1.42 (National Institutes of Health, Bethesda, MD, USA).

The hippocampal lesion volume ratio (\%) was determined by calculating the relative injury ratio (\%) after comparison with the non-injured left hippocampus.

\section{Behavioral testing}

Locomotor activity

Locomotor activity was measured for 30 minutes in a white open top acrylic box $(40 \times 40 \times 40 \mathrm{~cm})$ with an illumination intensity of 20 lux at the box floor level. The activity was automatically recorded using the video tracking system using SMART 3.0 software (Panlab/Harvard Apparatus, Holliston, MA, USA). The distance moved $(\mathrm{mm})$, time spent in the center (25\%), time spent in the area margins, and mean walking speed $(\mathrm{cm} / \mathrm{sec})$ were also evaluated.

\section{Barnes maze test}

The Barnes maze test was conducted as previously described with minor modifications..$^{20}$ The maze consisted of a white, acrylic, circular platform (91-cm diameter) with 20 equally spaced holes and a black acrylic escape box $(20 \times 5 \times 6 \mathrm{~cm})$ along the perimeter. The maze was surrounded by four spatial cues at the height of the maze.

Acquisition trials

Each mouse was trained for four acquisition trials per day over 3 days with an inter-trial interval of 10 to 15 minutes. Immediately prior to the first trial, the mouse was placed in the middle of the maze in a black-colored starting cylinder (10-cm diameter) and a buzzer ( 80 to $90 \mathrm{~dB}$ ) was turned on. After 10 seconds, the chamber was lifted, and the mouse was pre-trained to enter the escape box by guiding it to the escape box and remaining there for 2 minutes. Following the pre-training trial, the first trial was initiated.

At the beginning of each trial, the mouse was placed in the same starting chamber, and 10 seconds after the onset of a buzzer and light, the chamber was lifted, and the mouse was free to explore the maze. The trial ended when the mouse entered the goal tunnel or after 3 minutes into the trial. Immediately after the mouse entered the tunnel, the buzzer was turned off and the mouse could stay in the tunnel for 1 minute.

After each trial, the entire maze was cleaned with 70\% alcohol and rotated to eliminate any intra-maze cues. Trials were recorded using a video tracking system with SMART software 3.0.

Probe trial

During the probe trial, the escape tunnel leading to the target box was closed. The 90-second probe trial was conducted on day 6; mice could explore the maze and visit the target hole. The latency and distance traveled to reach the target hole for the first time were recorded.

\section{Passive-avoidance test}

Cognitive function was assessed using a computerized one-triallearning passive avoidance test (PAT; Panlab/Harvard Apparatus, Barcelona, Spain). The apparatus consisted of a square white compartment $(25 \times 25 \times 24 \mathrm{~cm})$ connected to an enclosed dark compartment $(13.5 \times 7.5 \times 7.5 \mathrm{~cm})$ by a motorized sliding door. On day 3 after $\mathrm{CCl}$, all mice received acquisition training. They were placed in the bright chamber; after 5 seconds the automatic door between the two chambers was opened. Once the mice entered the dark chamber, the door was closed and the mouse received a $1 \mathrm{~mA}$ foot shock for 2 seconds via the grid floor. After 
delivery of the foot shock, the mice were left in the dark compartment for another 10 seconds. One day or 5 days after acquisition training, the mice were tested for retention of the task. They were placed in the bright chamber of the apparatus, and after 5 seconds, the door was opened. Latency to enter the dark chamber was recorded to a maximum of 300 seconds. Animals that did not cross into the dark chamber during the testing period were assigned a latency of 300 seconds.

\section{Light/dark test}

The apparatus consisted of a black compartment $(20 \times 40 \times 40$ $\mathrm{cm})$ and a white compartment $(20 \times 40 \times 40 \mathrm{~cm})$ separated by a connecting gate $(5 \times 8 \mathrm{~cm})$. Each animal was individually placed at the center of the bright compartment (facing away from the door) and the following parameters were measured for $5 \mathrm{~min}$ utes: latency of the initial movement from the light to dark area (latency of transition), total number of transitions between light and dark areas, total time spent in the light area.

\section{Tail suspension test}

One week after $\mathrm{CCl}$, the mice were subjected to the tail suspension test, performed as previously described. ${ }^{21}$ Briefly, mice were individually suspended by the tail to a hook using adhesive tape and recorded by a video camera placed directly in front of the mice for subsequent analysis. The measured behavioral score was immobility during the entire 6-minute test session, using the video tracking system with the SMART software 3.0.

\section{Rotarod testing}

Animals were tested on a 7-cm diameter Rotarod treadmill (LE 8500; Panlab SL, Barcelona, Spain) elevated $50 \mathrm{~cm}$ above the bottom of the apparatus and attached to a motor to control speed. Briefly, mice were placed on the rotarod at a starting speed of 4 $\mathrm{rpm} / \mathrm{min}$ and acceleration of $0.5 \mathrm{rpm} / \mathrm{sec}$; the mice were subjected to three trials. In each trial, animals were scored for their latency to fall (in seconds) and speed on fall ( $\mathrm{m} / \mathrm{sec}$ ). Animals rested a minimum of 1 hour between trials to avoid fatigue. The average latency to fall for the three trials was used in analyses.

\section{Statistical analysis}

All data are presented as mean \pm standard error of the mean. Statistical evaluation of the data were performed using two-way analysis of variance (ANOVA) and post hoc Turkey's test. The Kaplan-Meier method was used for survival analysis. $\mathrm{P}<0.05$ was considered statistically significant. Data were analysed using the PASW/SPSSTM software, version 18 (IBM Inc., Chicago, USA) and GraphPad Prism 5.01 (GraphPad Prism Software, San Diego, CA, USA).

\section{RESULTS}

\section{Physiological variables}

Measures including body weight, body temperature during surgery, age, survival rates, and health assessment score are shown in Table 1. There was no significant difference in body weight, body temperature during surgery, or age between the sham and $2-\mathrm{mm} \mathrm{CCl}$ for both adult and pediatric groups. Weight gain (\%)

Table 1. Physiological parameters in adult and pediatric mice following controlled cortical impact

\begin{tabular}{|c|c|c|c|c|}
\hline & \multicolumn{2}{|c|}{ Adult mice } & \multicolumn{2}{|c|}{ Pediatric mice } \\
\hline & Sham & $2-m m$ & Sham & $2-m m$ \\
\hline Number & 29 & 35 & 39 & 46 \\
\hline Age (wk) & $9.89 \pm 2.23$ & $10.57 \pm 2.20$ & $3.37 \pm 0.23$ & $3.37 \pm 0.21$ \\
\hline Body weight (baseline, g) & $24.67 \pm 1.41$ & $24.61 \pm 1.11$ & $10.64 \pm 1.53$ & $10.60 \pm 1.06$ \\
\hline Body temperature ${ }^{\text {a) }}\left({ }^{\circ} \mathrm{C}\right)$ & $36.37 \pm 0.95$ & $36.33 \pm 0.95$ & $36.30 \pm 1.22$ & $36.60 \pm 0.97$ \\
\hline \multicolumn{5}{|l|}{ Weight gain (\% of baseline) } \\
\hline POD 1 & $94.52 \pm 4.17$ & $93.10 \pm 5.30$ & $94.62 \pm 9.99$ & $89.79 \pm 9.11$ \\
\hline POD 2 & $95.29 \pm 4.71$ & $90.56 \pm 7.07$ & $94.15 \pm 15.06$ & $89.52 \pm 14.19$ \\
\hline POD 3 & $96.90 \pm 5.16$ & $94.19 \pm 7.23$ & $98.74 \pm 17.28$ & $97.12 \pm 16.70$ \\
\hline POD 7 & $97.13 \pm 4.98$ & $96.21 \pm 4.37$ & $132.5 \pm 10.84^{* * *}$ & $129.2 \pm 17.04^{* * *}$ \\
\hline \multicolumn{5}{|c|}{ Health assessment score in surviving animals } \\
\hline POD 1 & $1.40 \pm 3.22$ & $2.12 \pm 1.63$ & $1.64 \pm 2.81$ & $3.28 \pm 0.66$ \\
\hline POD 2 & $0.60 \pm 0.12$ & $1.36 \pm 1.22$ & $1.57 \pm 2.32$ & $3.19 \pm 0.45$ \\
\hline POD 3 & $0.40 \pm 0.91$ & $1.04 \pm 1.65$ & $1.14 \pm 1.75$ & $1.46 \pm 2.73$ \\
\hline
\end{tabular}

POD, postoperative day.

${ }^{a)}$ Average rectal temperature during surgery.

${ }^{* * *} \mathrm{P}<0.001$. Asterisks indicate significant difference between adult mice and pediatric mice. 

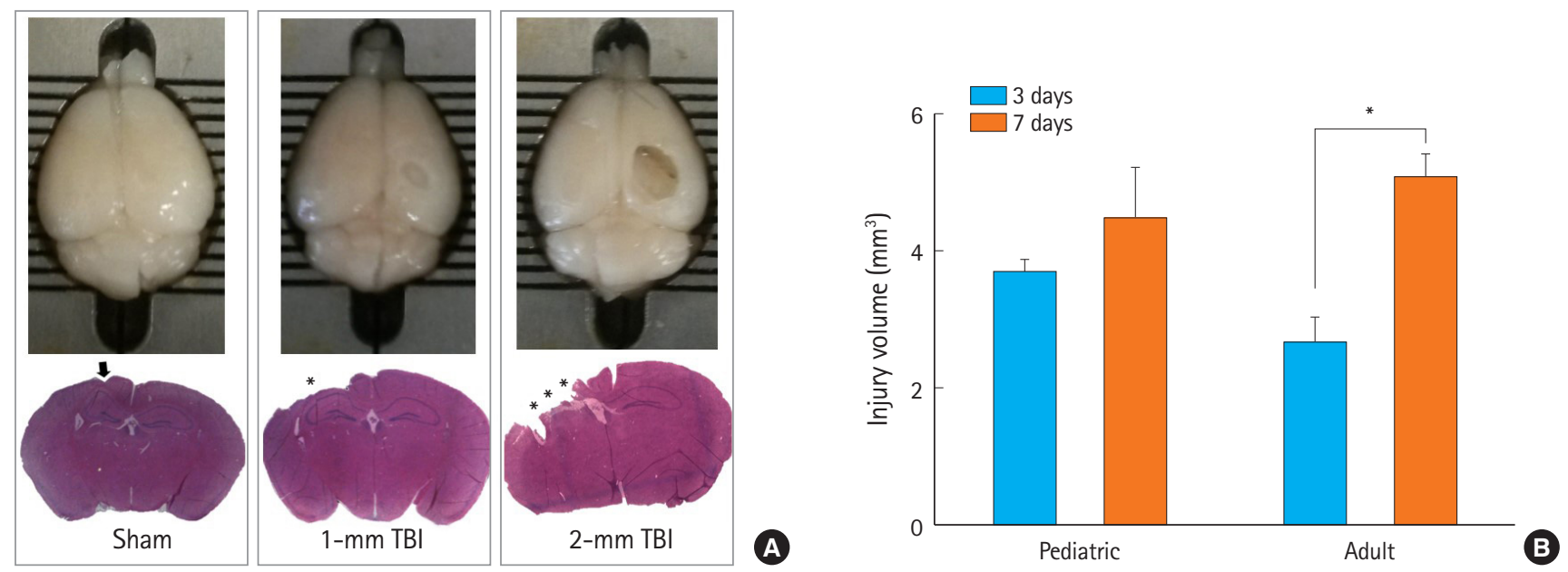

Fig. 3. Injury severity. (A) Gross examination of the brain shows significantly graded lesions. (B) Quantitative analysis of the injury volume following graded-controlled cortical impact showed a significant difference between injury and sham values via two-way ANOVA and post hoc Turkey's test $\left({ }^{*} \mathrm{P}<0.05,{ }^{* *} \mathrm{P}<0.001\right)$. There was a significant difference between 3 - and 7 -days after the 2 -mm depth of impact injury $\left({ }^{*} \mathrm{P}<0.01\right)$. The arrow indicates minimal injury due to drilling. $\mathrm{TBI}$, traumatic brain injury.

in the pediatric group was significantly different from that in the adult group on postoperative day 7 . However, there was no significant difference in the health assessment score during postoperative day 1 to 3 . Fig. 2 shows that the survival rate after surgery among pediatric mice was lower than that in the adult mice. The difference in survival rate was not surprising because pediatric mice during the weaning period were young \& small, and may be more sensitive to surgical complications.

\section{Cortical impact injury volumes}

To determine injury severity according to the depth of impact, we first impacted the mouse cortex with three different depths of impact (0.0, 1.0, and $2.0 \mathrm{~mm}$ ). Analysis of the tissue samples obtained 7 days after injury demonstrated a progressively larger lesion of the brain proportional to the depth of impact. Sham mice showed no or minimal injury due to drilling. All mice with 2-mm depth of impact showed apparent injury to the hippocampus, in marked contrast to mice with 1-mm depth of impact that had an intact hippocampus, as seen on H\&E staining (Fig. 3A). Therefore, we used a 2-mm depth of impact (severe and moderate injury) to analyze differences between adult and pediatric groups. The procedure is presented in Fig. 3.

In the measurement of the injury volume of the cortex and hippocampus in adult and pediatric mice, a statistically significant difference was not observed between the adult and pediatric male mice at 3 and 7 days following 2-mm depth of impact $\mathrm{CCl}$ (Fig. 3B). However, there was a difference in the injury volume between 3 and 7 days after $\mathrm{CCl}$ in the adult groups (Fig. 3B).

\section{Neurobehavioral outcomes}

Spatial learning and memory deficits in the Barnes maze

Acquisition phase: During training, there was a significant difference in latency to enter the target hole between sham and 2-mm TBI adult mice. Latency significantly decreased during the three training days, indicating a mean performance improvement in sham adult mice. Interestingly, pediatric mice did not show a significant difference in latency or latency decrease between the sham and 2-mm CCl groups during training (Fig. 4A).

The distance $(\mathrm{cm})$ travelled (Fig. 4B) to enter the target hole and mean total number of errors (Fig. $4 \mathrm{C}$ ), and average speed (Fig. 4D) were analyzed using a repeated measures ANOVA; these values were found to significantly decrease during the acquisition phase in the sham adult mice but not in the $2-\mathrm{mm} \mathrm{CCl}$ adult mice. In contrast, pediatric mice did not show a significant decrease in the distance $(\mathrm{cm})$ travelled or the mean total number of errors during training. Unlike the pediatric group, the adult group exhibited a significantly higher average moving speed during training in sham rather than 2-mm CCl mice.

Retention phase: An unpaired t-test showed significant differences in the primary latency to the target hole and to adjacent holes between the pediatric and adult sham mice and between the sham and 2-mm CCl adult mice, indicating that the adult 2-mm TBI mice had memory deficits (Fig. 4E). However, the primary path length to the target hole was not significantly different between the groups (Fig. 4F).

\section{Passive avoidance performance}

Regardless of $\mathrm{TB}$, pediatric and adult mice demonstrated a good 

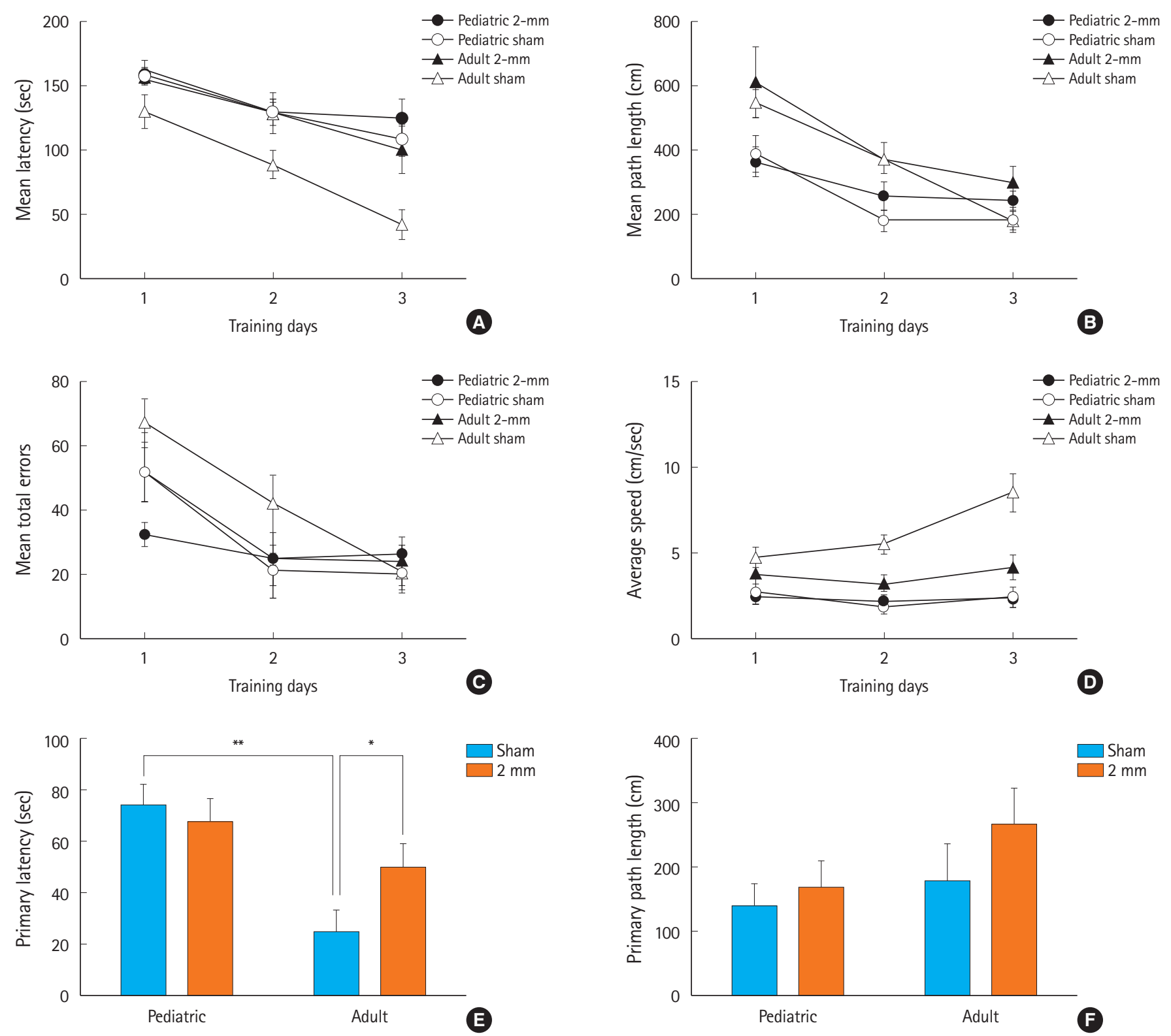

Fig. 4. Spatial learning and memory in animals following traumatic brain injury. The Barnes maze was used to determine latencies and distances, errors, and speed to find the escape box. (A) Mean latency (second), (B) mean path length (cm), (C) mean total errors, and (D) average speed (cm/sec) in acquisition trials; (E) mean latency (second) and (F) mean path length $(\mathrm{cm})$ on day 5 of retention trials. Asterisks indicate level of significance of difference. ${ }^{*} \mathrm{P}<0.05$. ${ }^{* *} \mathrm{P}<0.01$. $n=10-12$ per group; bars and whiskers represent mean \pm standard error of the mean.

ability to learn the task as they avoided coming into the chamber in which they were given a shock on the previous day and 4 days ago. The step-through latency was not significantly different between the groups (Fig. 5).

\section{Locomotor activity in the open-field test}

The overall locomotion activity was not influenced by $2-\mathrm{mm}$ TBI in both the pediatric and adult groups (Fig. 6A), indicating that 2-mm CCl did not have deleterious consequences on motor behavior during the 7 days after $\mathrm{CCl}$ (Fig. 6).

\section{Anxiety-like behavior}

In the light-dark box test, both pediatric and adult mice after 2-mm CCl did not show a significant difference in the initial latency of transition (Fig. 7A), time spent in the light compartment (Fig. 7B), or transition times (Fig. 7C) from sham mice. Interestingly, adult mice spent significantly more time in the light compartment than pediatric mice. Notably, 2-mm CCl did not also lead to a significant reduction in immobility time relative to sham mice in both the pediatric and adult groups in the tail-suspension test (Fig. 7D). 


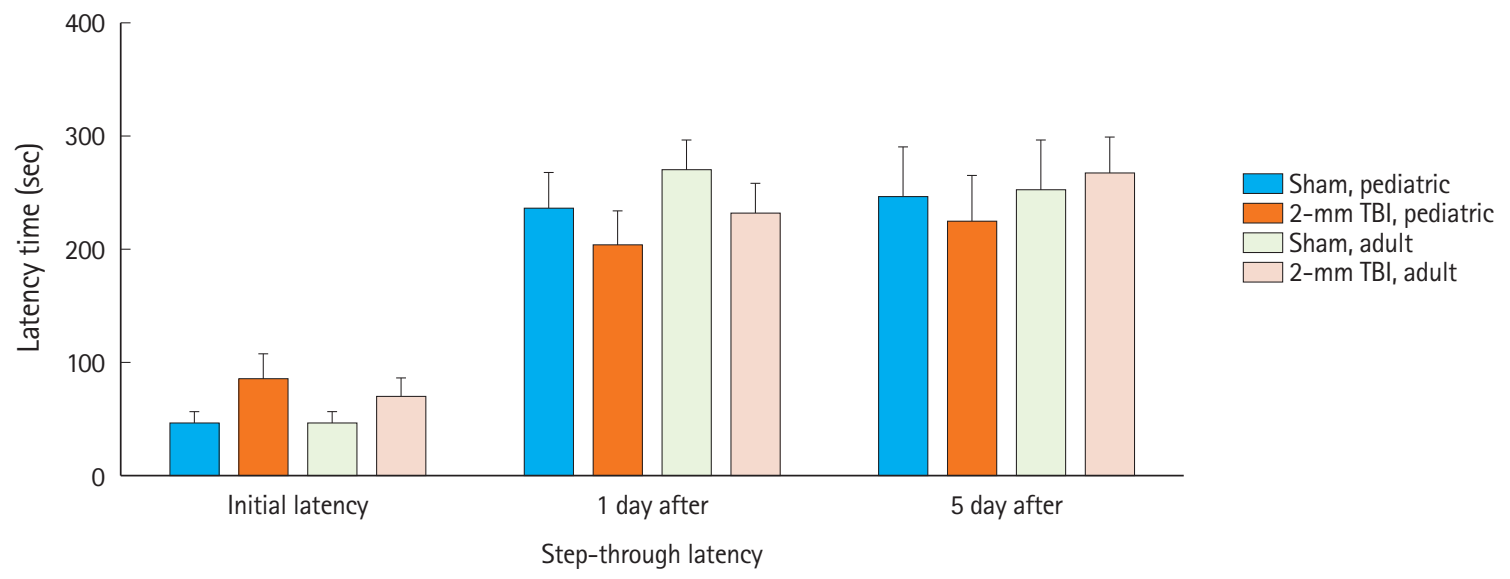

Fig. 5. Effects of traumatic brain injury (TBI) on crossover latency in the retention trial of the passive avoidance test. When animals were given aversive stimuli 2 days after injury and tested for their ability to remember stimuli 1 and 5 days after the stimuli, controlled cortical impact mice were not significantly impaired in their recall ability.
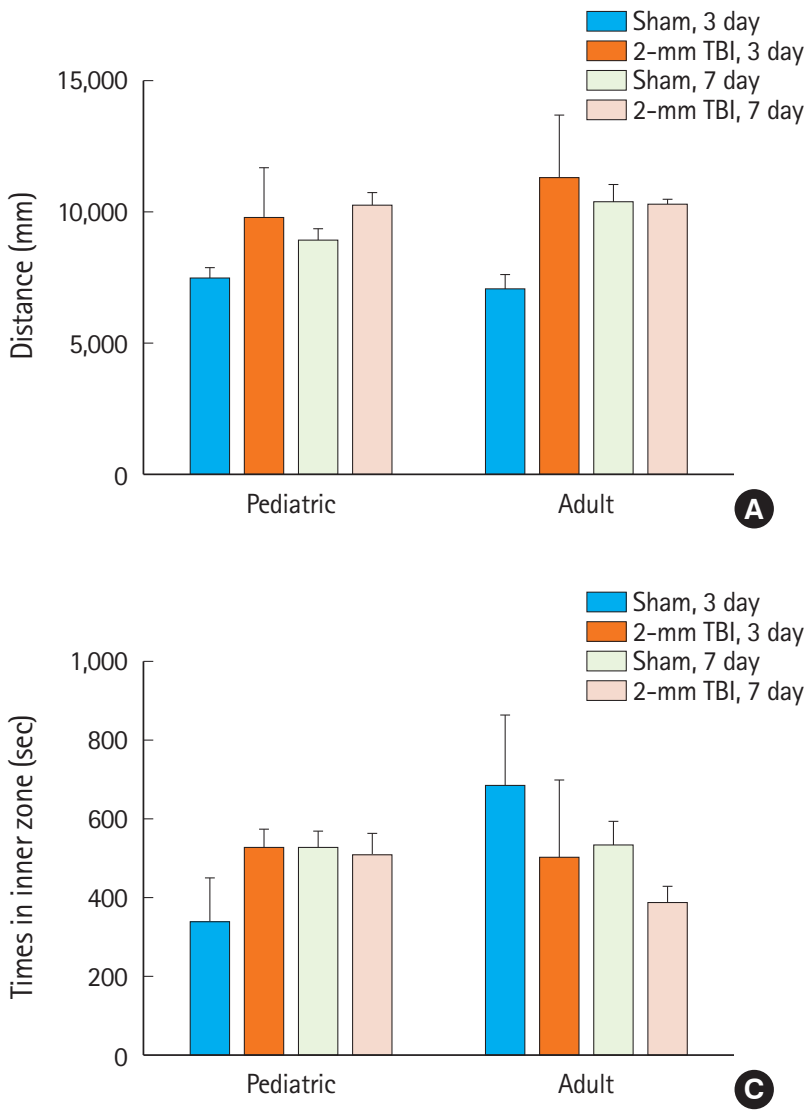
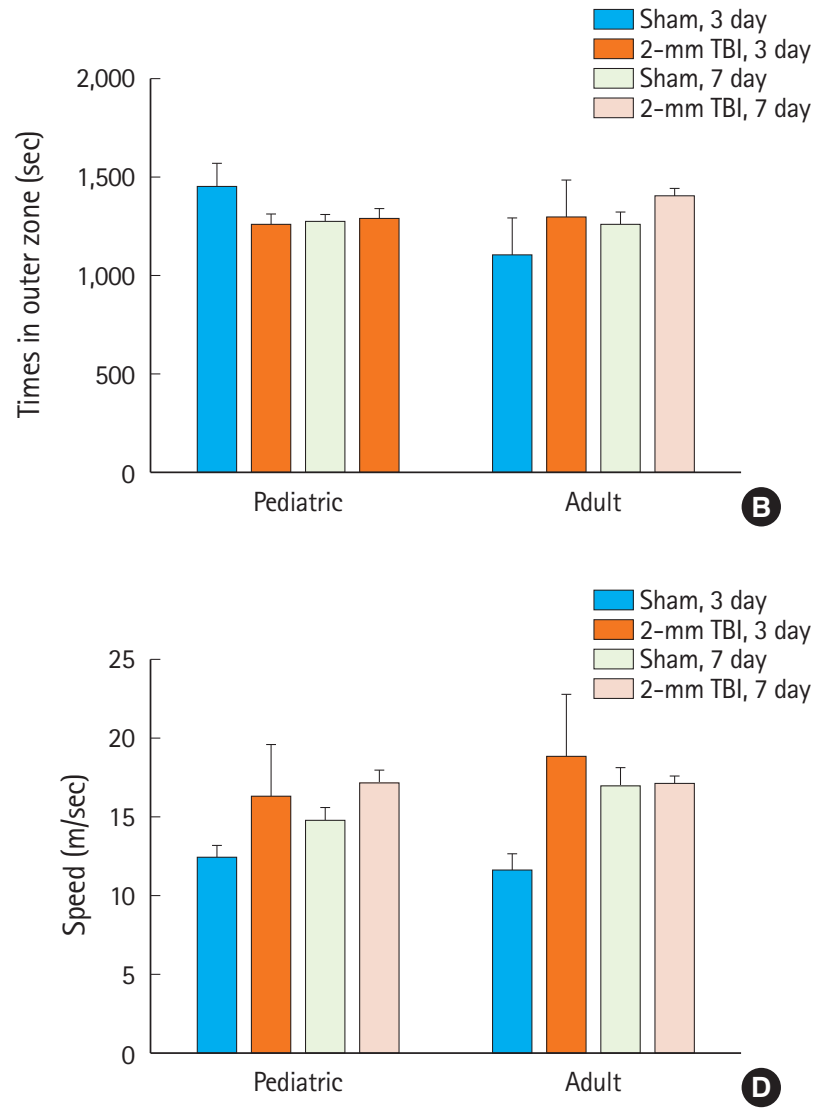

Fig. 6. Effects of traumatic brain injury (TBI) on locomotor activity in pediatric and young adult mice. The typical running pathway record generated by the tracking software shows the search paths and strategies of each group of mice. (A) Total distance moved; (B) distance moved in the border field; (C) distance moved in the central field; (D) mean speed. Data were analyzed using a two-way analysis of variance, followed by Tukey's post hoc test. $n=10-$ 12 per group; bars and whiskers represent mean \pm standard error of the mean.

\section{Motor performance}

There was a significant difference between the adult and pediatric $2-\mathrm{mm} \mathrm{CCl}$ at 3 days, in the performance time and falling ve- locity when evaluating motor performance using the accelerating rotarod $(P<0.05)$. However, no significant differences were observed between the sham and 2-mm TBI mice in each age group 

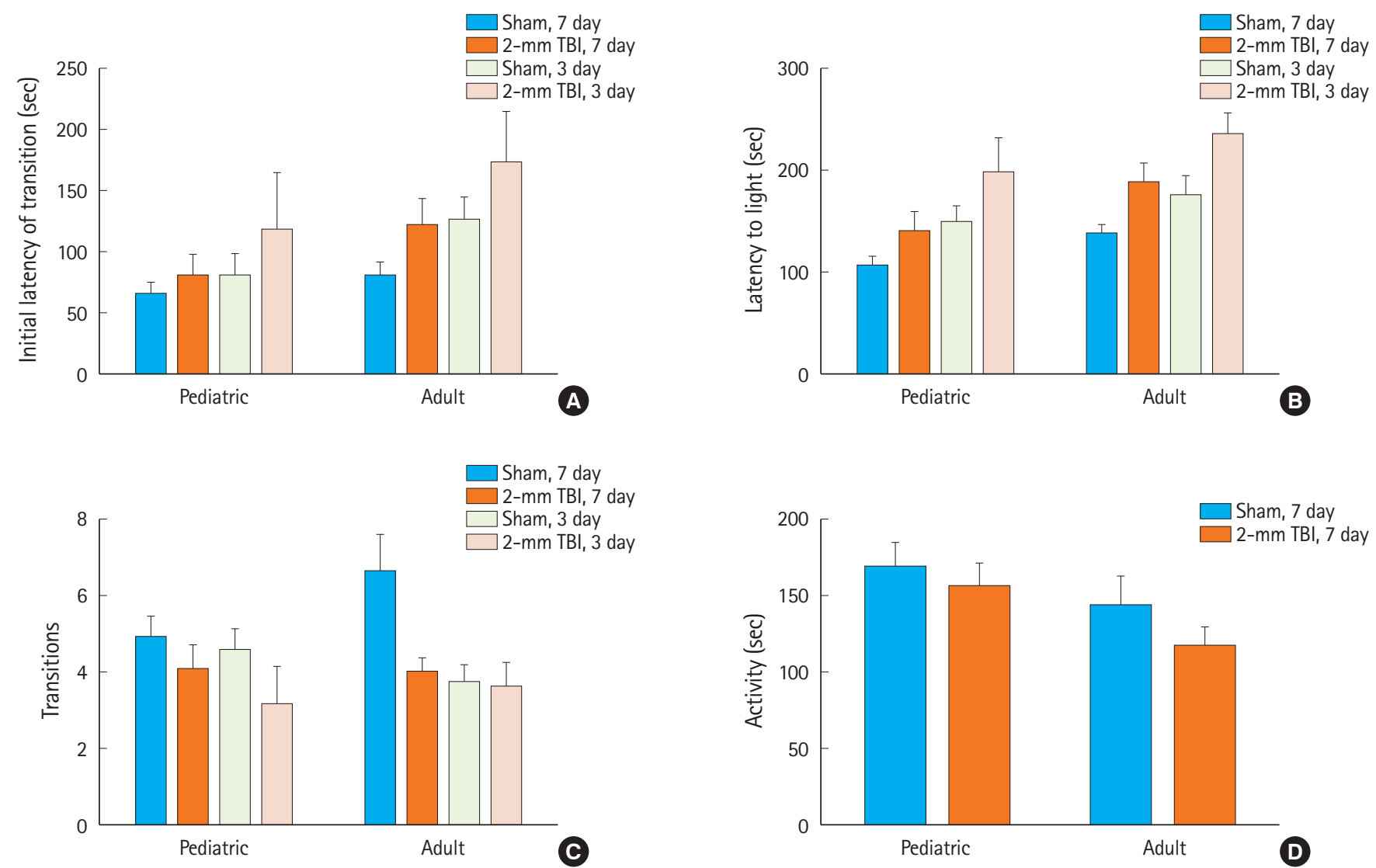

Fig. 7. Anxiety-like behavior in animals following traumatic brain injury (TBI). (A-C) Light/dark preference test and (D) tail suspension test. There was no significant difference in the initial latency of transition, time spent in the light compartment, times of transitions in the light-dark box, or activity times. Data are expressed as mean \pm standard error of the mean ( $n=6-8$ per group).
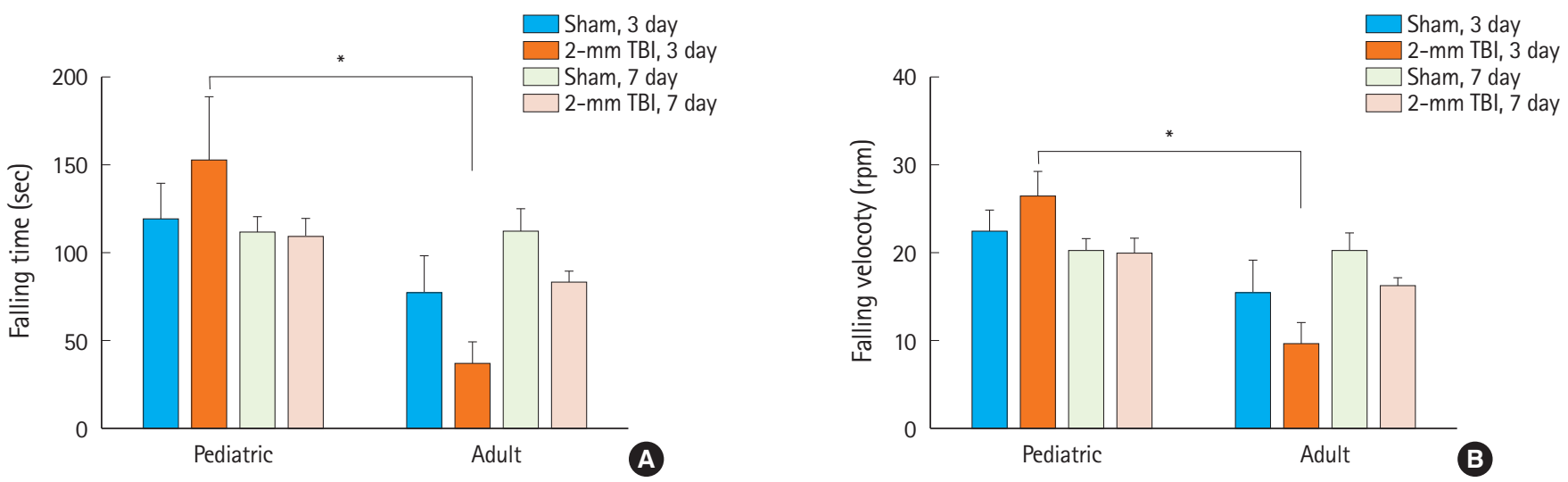

Fig. 8. Motor performance of animals following traumatic brain injury (TBI). (A) Falling time (second) and (B) falling velocity (rpm). Data are expressed as mean \pm standard error of the mean. $n=8-10$ per group and were analyzed using a two-way analysis of variance, followed by Tukey's post hoc test. Asterisks indicate level of statistical significance. ${ }^{*} \mathrm{P}<0.05$.

for the performance time and falling velocity when using the accelerating rotarod (Fig. 8).

\section{DISCUSSION}

In addition to analyzing mortality rates and physiological variables, this study characterized histopathological responses and 
neurobehavioral outcomes in pediatric and adult mice following $\mathrm{CCl}$. Pediatric mice had a higher mortality rate than young adult mice following $\mathrm{CCl}$. Although there are similarities between head injury in the developing and adult brain, pediatric brain injury poses unique challenges. ${ }^{22}$ The primary injury is generally more diffuse in children because of a greater head-to-torso ratio and brain-water content. ${ }^{22}$ Clinical studies have demonstrated that the developing brain may also be more vulnerable to traumatic injury than the adult brain. ${ }^{22-24}$ However, in our study, we applied the same impact for pediatric and adult brains.

Compared with other TBI models, such as the fluid percussion brain injury blast wave and weight drop, the $\mathrm{CCl}$ model is an easy and accurate experimental TBI research tool that uses a mechanical system to deliver physical impact to the exposed mouse dura. ${ }^{25-27}$ It can precisely control many injury parameters, such as velocity, position, area, time, and depth of impact, accurately producing nearly identical injuries across groups of mice. ${ }^{26}$ Therefore, the $\mathrm{CCl}$ model can overcome the epidemiological complexity and age-related inconsistent clinical findings seen in human TBIs.

In this study, the $\mathrm{CCl}$ was performed over the right parietal cortex and near the middle portions of the bregma, lambda, and sagittal suture. Thus, hippocampal injury, ranging from mild to severe, similar to that occurring in humans, could be observed. In a pilot experiment, after mild 1.0-mm impact in adult male mice, there appeared to be no apparent injury to the hippocampus with limited cortical lesions. However, a severe 2.0-mm impact evoked hippocampal injury with a graded pattern. Our graded injury pattern is similar to that reported by other studies. ${ }^{28-30}$ This histopathological graded injury in the cortex and hippocampal area is closely related to differential behavioral and functional findings. ${ }^{31,32}$ Some experimental models, such as stroke, ${ }^{33,34}$ cardiac arrest, ${ }^{16}$ and $\mathrm{TB} \mathrm{I}^{35,36}$ models, have suggested age-dependent differences in neuronal ischemic vulnerability. Although the mechanisms have not been fully investigated, numerous neurochemical and physiological changes at different ages could play an important role in the responsiveness to various ischemic insults, which leads to an age-dependent discrepancy in excitotoxicity, cerebral edema, oxidative stress, inflammatory response, and blood-brain barrier permeability after brain injury. ${ }^{35,37-39}$ An important observation in the present series of histological investigations is that adult mice showed significantly more similar degree of histological injury at the acute stage compared with pediatric mice. However, there was a significant difference in the magnitude of injury volume between 3- and 7-days after adult TBI.

Although the influence of age differences on pathophysiological processes after TBI have not been completely investigated, TBI has a complex pathophysiological process consisting of several overlapping phases, including primary injury, secondary injury, and regeneration. After the primary injury, such as focal hematomas, contusions, or diffuse injury, secondary injury mechanisms are initiated that manifest over a period of hours to days through complex biochemical and physiological processes. This secondary injury could be exacerbated by the aging process. ${ }^{40,41}$

Severe TBI presents significant cognitive and/or affective dysfunction that can have perpetual adverse consequences on the quality of life. Cognitive problems following TBI include decreased memory and learning abilities, impaired attention and concentration, reduced processing speed, word finding difficulties, and impaired executive functioning (i.e., reduced ability to plan, sequence, prioritize, think flexibly, abstract, or problem solve).? The cognitive processes that result in memory impairment following $\mathrm{TBI}$ are not fully understood. Although experimental animal studies have demonstrated that TBI induces both retrograde and anterograde memory deficits in tests such as the Morris Water maze, it is uncertain as to what types of learning abilities or memory are affected following $\mathrm{TBI}$, and which anatomical structures are responsible for the observed deficits. ${ }^{42}$ Our study demonstrated that the Barnes circular maze was an efficient cognitive task to assess spatial/non-spatial learning following $\mathrm{CCl}$ injury in adult mice, but it was impossible to assess spatial learning impairment in pediatric mice.

We used the PAT to evaluate amygdala-dependent emotional memory after TBI. Passive avoidance performance is an adaptive response to a stressful experience that serves as a measure of learning and memory. This test is based on the innate preference of mice for the dark compartment of the apparatus, and suppression of this innate preference following exposure to an inescapable shock involves NMDA receptors in the amygdala and hippocampus in the formation of post-training memory. ${ }^{43}$ However, the PAT in our study did not show significant memory impairment after 2-mm focal TBI of the right parietal lobe in both pediatric and adult mice. Khalin et al. ${ }^{44}$ showed that with a weight-drop closed head injury, similar results were achieved in mice with TBI from both experimental groups on days 1 and 4. However, on day 7 , the mean latency was significantly shorter in mice with TBI caused by $250 \mathrm{~g}$ of falling weight than in sham-operated mice and mice with TBI caused by $200 \mathrm{~g}$ of falling weight. This indicates that at 1 week after injury, mice with TBI caused by $250 \mathrm{~g}$ of falling weight developed cognitive deficits. Further long-term studies are warranted to clarify this observation.

In addition to cognitive changes that occur following TBI, most individuals with TBI are diagnosed with one or more newly acquired psychiatric diagnoses, such as anxiety-like behavior and depression, after injur. ${ }^{45}$ 
A growing body of experimental evidence indicates that postTBI anxiety disorders do not show a clear association with injury severity, although they appear to be more prevalent following mild $\mathrm{TBI}$, unlike cognitive impairments that are worsened with an increased severity of injur.. ${ }^{46}$ Evaluation of anxiety states by using the open-field test, light/dark test, and tail suspension test in our study did not show definite differences in anxiety-like behavior between sham and 2-mm focal TBI on the right parietal lobe in both pediatric and adult mice.

The study has several limitations. First, we did not evaluate female mice in our study. Second, we did not test other strains of mice. Third, we lesioned the focal right parietal lobe; we need to further investigate other types of focal and diffuse brain injuries. Therefore, extensive testing on behavioral and electrophysiological outcomes is necessary to evaluate the sex effect in detail. Fourth, we did not administer a 2.5- or 3.0-mm impact because of its high mortality rate after surgery. Fifth, we used tiletamine/ zolazepam for anesthesia, which is known to block NMDA receptors. ${ }^{47}$ Hence, the anesthetic could affect the result of the neurobehavioral tests such as the PAT. Finally, mice were sacrificed 3 and 7 days after injury; thus, the long-term effects (more than 2 months) on histopathological outcomes were not assessed.

We demonstrated age-dependent differences in the loss of cortical mantle volume and neurobehavioral outcomes at an acute stage following $\mathrm{CCl}$. There were age-dependent differences in the rate of injury progression following severe impact and spatial memory deficits in the experimental TBI models. Pediatric mice demonstrated faster injury progression than adult mice, and adult mice exhibited spatial memory deficits following $\mathrm{CCl}$. Understanding these differences could be an important first step in research on the age-dependent progression of the pathogenesis and developing age appropriate therapeutics for TBI.

\section{CONFLICT OF INTEREST}

No potential conflict of interest relevant to this article was reported.

\section{ACKNOWLEDGMENTS}

This research was supported by the Basic Science Research Program through the National Research Foundation of Korea funded by the Ministry of Education, Science and Technology (2015R1A2A2A01002303 \& NRF-2016R1D1A1B04930247).

\section{REFERENCES}

1. McHugh GS, Engel DC, Butcher I, et al. Prognostic value of secondary insults in traumatic brain injury: results from the IMPACT study. J Neurotrauma 2007;24:287-93.

2. Rincon F, Kang J, Vibbert M, Urtecho J, Athar MK, Jallo J. Significance of arterial hyperoxia and relationship with case fatality in traumatic brain injury: a multicentre cohort study. J Neurol Neurosurg Psychiatry 2014;85:799-805.

3. Coronado VG, Xu L, Basavaraju SV, et al. Surveillance for traumatic brain injury-related deaths: United States, 1997-2007. MMWR Surveill Summ 2011;60:1-32.

4. Centers for Disease Control and Prevention (CDC). CDC grand rounds: reducing severe traumatic brain injury in the United States. MMWR Morb Mortal Wkly Rep 2013;62:549-52.

5. Appelboom G, Zoller SD, Piazza MA, et al. Traumatic brain injury in pediatric patients: evidence for the effectiveness of decompressive surgery. Neurosurg Focus 2011;31:E5.

6. Riggio S. Traumatic brain injury and its neurobehavioral sequelae. Neurol Clin 2011;29:35-47.

7. Ashman TA, Gordon WA, Cantor JB, Hibbard MR. Neurobehavioral consequences of traumatic brain injury. Mt Sinai J Med 2006;73:999-1005.

8. Castano Monsalve $B$, Laxe $S$, Bernabeu Guitart $M$, Vilarrasa $A B$, Quemada Jl. Behavioral scales used in severe and moderate traumatic brain injury. NeuroRehabilitation 2014;35:67-76.

9. McAllister TW. Neurobehavioral sequelae of traumatic brain injury: evaluation and management. World Psychiatry 2008;7:310.

10. Babikian T, Prins ML, Cai Y, et al. Molecular and physiological responses to juvenile traumatic brain injury: focus on growth and metabolism. Dev Neurosci 2010;32:431-41.

11. Kannan N, Ramaiah R, Vavilala MS. Pediatric neurotrauma. Int J Crit IIIn Inj Sci 2014;4:131-7.

12. Gomez PA, Lobato RD, Boto GR, De la Lama A, Gonzalez PJ, de la Cruz J. Age and outcome after severe head injury. Acta Neurochir (Wien) 2000;142:373-80.

13. Narayan RK, Greenberg RP, Miller JD, et al. Improved confidence of outcome prediction in severe head injury. A comparative analysis of the clinical examination, multimodality evoked potentials, CT scanning, and intracranial pressure. J Neurosurg 1981;54:751-62.

14. MRC CRASH Trial Collaborators, Perel $P$, Arango $M$, et al. Predicting outcome after traumatic brain injury: practical prognostic models based on large cohort of international patients. BMJ 2008;336:425-9.

15. Hukkelhoven CW, Steyerberg EW, Rampen AJ, et al. Patient 
age and outcome following severe traumatic brain injury: an analysis of 5600 patients. J Neurosurg 2003;99:666-73.

16. Deng $G$, Yonchek JC, Quillinan $N$, et al. A novel mouse model of pediatric cardiac arrest and cardiopulmonary resuscitation reveals age-dependent neuronal sensitivities to ischemic injury. J Neurosci Methods 2014;222:34-41.

17. Xiong Y, Mahmood A, Chopp M. Animal models of traumatic brain injury. Nat Rev Neurosci 2013;14:128-42.

18. Kosaka Y, Quillinan N, Bond C, Traystman R, Hurn P, Herson P. GPER1/GPR30 activation improves neuronal survival following global cerebral ischemia induced by cardiac arrest in mice. Transl Stroke Res 2012;3:500-7.

19. Allen $D$, Nakayama $S$, Kuroiwa $M$, et al. SK2 channels are neuroprotective for ischemia-induced neuronal cell death. J Cereb Blood Flow Metab 2011;31:2302-12.

20. Patil SS, Sunyer B, Hoger $H$, Lubec $G$. Evaluation of spatial memory of $C 57 B L / 6 J$ and $C D 1$ mice in the Barnes maze, the Multiple T-maze and in the Morris water maze. Behav Brain Res 2009;198:58-68.

21. Cryan JF, Kelly PH, Neijt HC, Sansig G, Flor PJ, van Der Putten H. Antidepressant and anxiolytic-like effects in mice lacking the group III metabotropic glutamate receptor mGluR7. Eur J Neurosci 2003;17:2409-17.

22. Potts $\mathrm{MB}$, Koh $\mathrm{SE}$, Whetstone $W D$, et al. Traumatic injury to the immature brain: inflammation, oxidative injury, and ironmediated damage as potential therapeutic targets. NeuroRx 2006;3:143-53.

23. Adelson PD, Clyde B, Kochanek PM, Wisniewski SR, Marion DW, Yonas H. Cerebrovascular response in infants and young children following severe traumatic brain injury: a preliminary report. Pediatr Neurosurg 1997;26:200-7.

24. Vigil-Sewell J, Sargent JD. Long-term outcome after severe brain injury in preschoolers is worse than expected. Arch Pediatr Adolesc Med 1996;150:228.

25. Chen $Y$, Mao H, Yang KH, Abel T, Meaney DF. A modified controlled cortical impact technique to model mild traumatic brain injury mechanics in mice. Front Neurol 2014;5:100.

26. Romine J, Gao X, Chen J. Controlled cortical impact model for traumatic brain injury. J Vis Exp 2014;90:e51781.

27. Clark RS, Schiding JK, Kaczorowski SL, Marion DW, Kochanek PM. Neutrophil accumulation after traumatic brain injury in rats: comparison of weight drop and controlled cortical impact models. J Neurotrauma 1994;11:499-506.

28. Budinich CS, Chen H, Lowe D, Rosenberger JG, Bernstock JD, McCabe JT. Mouse brain PSA-NCAM levels are altered by graded-controlled cortical impact injury. Neural Plast 2012; 2012:378307.
29. Janz DR, Hollenbeck RD, Pollock JS, McPherson JA, Rice TW. Hyperoxia is associated with increased mortality in patients treated with mild therapeutic hypothermia after sudden cardiac arrest. Crit Care Med 2012;40:3135-9.

30. Brody DL, Mac Donald C, Kessens CC, et al. Electromagnetic controlled cortical impact device for precise, graded experimental traumatic brain injury. J Neurotrauma 2007;24:65773.

31. Saatman KE, Feeko KJ, Pape RL, Raghupathi R. Differential behavioral and histopathological responses to graded cortical impact injury in mice. J Neurotrauma 2006;23:1241-53.

32. Adelson PD, Fellows-Mayle W, Kochanek PM, Dixon CE. Morris water maze function and histologic characterization of two age-at-injury experimental models of controlled cortical impact in the immature rat. Childs Nerv Syst 2013;29:43-53.

33. Liu F, McCullough LD. Interactions between age, sex, and hormones in experimental ischemic stroke. Neurochem Int 2012; 61:1255-65.

34. Cheng J, Hu W, Toung TJ, et al. Age-dependent effects of testosterone in experimental stroke. J Cereb Blood Flow Metab 2009;29:486-94.

35. Claus CP, Tsuru-Aoyagi K, Adwanikar H, et al. Age is a determinant of leukocyte infiltration and loss of cortical volume after traumatic brain injury. Dev Neurosci 2010;32:454-65.

36. Duhaime AC, Raghupathi R. Age-specific therapy for traumatic injury of the immature brain: experimental approaches. Exp Toxicol Pathol 1999;51:172-7.

37. Davis M, Mendelow AD, Perry RH, Chambers IR, James OF. The effect of age on cerebral oedema, cerebral infarction and neuroprotective potential in experimental occlusive stroke. Acta Neurochir Suppl (Wien) 1994;60:282-4.

38. Leitgeb J, Mauritz W, Brazinova A, et al. Effects of gender on outcomes after traumatic brain injury. J Trauma 2011;71:1620-6.

39. Titus DJ, Furones $C$, Kang Y, Atkins CM. Age-dependent alterations in CAMP signaling contribute to synaptic plasticity deficits following traumatic brain injury. Neuroscience 2013;231: 182-94.

40. Cernak I. Animal models of head trauma. NeuroRx 2005;2: 410-22.

41. Hawthorne C, Piper I. Monitoring of intracranial pressure in patients with traumatic brain injury. Front Neurol 2014;5:121.

42. Whiting MD, Baranova Al, Hamm RJ. Cognitive impairment following traumatic brain injury. In: Levin ED, Buccafusco JJ, editors. Animal models of cognitive impairment. Boca Raton, FL: CRC Press; 2006;301-14.

43. Gacar N, Mutlu O, Utkan T, Komsuoglu Celikyurt I, Gocmez SS, Ulak G. Beneficial effects of resveratrol on scopolamine but 
not mecamylamine induced memory impairment in the passive avoidance and Morris water maze tests in rats. Pharmacol Biochem Behav 2011;99:316-23.

44. Khalin I, Jamari NL, Razak NB, et al. A mouse model of weight-drop closed head injury: emphasis on cognitive and neurological deficiency. Neural Regen Res 2016;11:630-5.

45. Wood RL, Worthington A. Neurobehavioral abnormalities associated with executive dysfunction after traumatic brain injury. Front Behav Neurosci 2017;11:195.
46. Siopi $E$, Llufriu-Daben $G$, Fanucchi F, Plotkine M, Marchand-

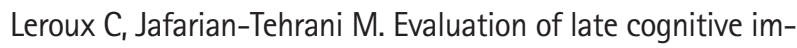
pairment and anxiety states following traumatic brain injury in mice: the effect of minocycline. Neurosci Lett 2012;511:1105.

47. Popik P, Holuj M, Kos T, Nowak G, Librowski T, Salat K. Comparison of the Psychopharmacological effects of tiletamine and ketamine in rodents. Neurotox Res 2017;32:544-54. 